\title{
Pflanzliche Sekundärstoffe: Ethnobotanische Überlegungen zu ihrer Bedeutung für die menschliche Ernährung und die Medizin - Teil 1: Grundlagen der pflanzlichen Sekundärstoffe
}

\author{
Rainer Nowack \\ Lindau, Deutschland
}

Schlüsselwörter

Sekundärstoffe · Cytochrom-P450-Enzyme, CYP · Koevolution

\section{Zusammenfassung}

Die sogenannten Sekundärstoffe der Pflanzen haben spezifische Funktionen für den pflanzlichen Organismus und seine ökologische Position, z.B. zur Abwehr von Herbivoren oder zur Attraktion bestäubender Insekten. Herbivoren einschliesslich des Menschen haben infolge von Koevolution mit den Pflanzen Transportproteine, Enzyme und weitere Instrumente erworben, um einerseits geeignete Sekundärstoffe zu resorbieren, zu metabolisieren oder für eigene Zwecke zu instrumentalisieren und andererseits toxische Sekundärstoffe zu meiden, nach Resorption auszuscheiden oder zu detoxifizieren.

(c) 2016 S. Karger GmbH, Freiburg
Keywords

Secondary plant compounds - Cytochrome P450 enzyme, CYP . Coevolution

\section{Summary}

Secondary Plant Compounds: Ethnobotanical Reflections Regarding Their Role in Human Nutrition and in Medicine - Part 1: Principles of Secondary Plant Compounds

Secondary plant compounds have specific functions within the plants and for their ecologic positions, e.g. for deterring predators or for attracting pollinating insects. Herbivorous animals as well as human beings dealing with these compounds have acquired mechanisms throughout their coevolution with plants to resorb and utilize favorable compounds and to avoid toxic compounds or inactivate them after accidental ingestion.

\section{Nahrung und/oder Medizin: Entscheiden die pflanzlichen Sekundärstoffe über die unterschiedlichen Karrieren von Pflanzen im Dienste des Menschen?}

Pflanzliche Sekundärstoffe bestimmen die Wirksamkeit von traditionellen pflanzlichen Therapeutika. Sie sind die Basis für die meisten Moleküle in pharmazeutischen Präparaten und ihr Gehalt wird in Nahrungsergänzungsmitteln oder in «Functional Food» ausgelobt. Für pflanzliche Nahrung, wie sie in der westlichen Zivilisation zur

Diese Arbeit und zwei Fortsetzungen basieren auf einer Abschlussarbeit des Zertifikatsstudiengangs (CAS) «Ethnobotanik und Ethnomedizin» der Universität Zürich, 2014 (Betreuerin: Dr. Caroline Weckerle, Institut für Systematische Botanik, Universität Zürich, Schweiz).
Deckung des täglichen Energiebedarfs konsumiert wird, spielt der Gehalt an Sekundärstoffen dagegen nur eine unwesentliche Rolle.

Das hippokratische Diktum «Nahrung sollte auch unsere Medizin sein» scheint nicht mehr gültig zu sein. Im Gegenteil: Es hat eine deutliche Trennung von Nahrungsund Medizinalpflanzen stattgefunden.

In diesem und zwei weiteren Beiträgen wird am Beispiel zahlreicher Pflanzen untersucht, wie sich diese Trennung im Verlauf der Zivilisationsgeschichte vollzogen hat und welche Rolle die Sekundärstoffe dabei hatten. Speziell geht es um die Frage, ob sich bereits die wilden Vorläufer der Nahrungspflanzen so grundsätzlich von den übrigen und speziell von den späteren Arzneipflanzen unterschieden, dass sich die Entwicklungen sozusagen zwangsläufig trennen mussten.

\section{KARGER}

Fax +497614520714 Information@Karger.com www.karger.com

\section{(๑) 2016 S. Karger GmbH, Freiburg}

$1015-0684 / 16 / 0283-0157 \$ 39.50 / 0$

www.karger.com/szg
Prof. Dr. med. Rainer Nowack

Dennenmoos 26, 88131 Lindau, Deutschland nowack@dialyse-lindau.de 
In diesem ersten Beitrag werden die Funktionen der Sekundärstoffe in den Pflanzen und ihre Bedeutung für die ökologische Position vorgestellt. Für die Tierwelt stellen die Sekundärstoffe eine Herausforderung dar, der sie mit im Verlauf der Evolution erworbenen Mechanismen $\mathrm{zu}$ ihrer Inaktivierung und Instrumentalisierung begegnen.

Beim Menschen definieren diese Instrumente zur Metabolisierung und Utilisation von Sekundärstoffen das Spektrum der für ihn als Nahrung geeigneten Pflanzen. Ein Verständnis dieser metabolischen Grundkondition ist unerlässlich, um einordnen zu können, nach welchen Kriterien der Mensch seit dem Neolithikum Pflanzen zur Domestikation ausgewählt hat und nach welchem Muster er überhaupt Pflanzen verwendet.

\section{Der Mensch als Herbivor}

Zum Zeitpunkt ihrer Abspaltung von den Schimpansen vor 4,6-6,2 Millionen Jahren lebten die waldbewohnenden frühen Hominiden (Ardipithecus ramidus) von einer breiten Vielfalt pflanzlicher Kost [1]. Vor 4 Millionen Jahren begann Australopithecus als Vorläufer von Homo offene Landschaften zu besiedeln. Nun dominierte eine harte, auf die Zähne abrasiv wirkende Pflanzennahrung, die vorwiegend von Samen der Savannengräser stammte. Ablesbar ist diese Umstellung unter anderem an den sich vergrössernden Zähnen, der Verstärkung des Zahnschmelzes und an Veränderungen der Kiefermuskulatur [2].

Die Gattung Homo erlernte vor 2,5 Millionen Jahren die Nutzung von Werkzeugen und des Feuers. Pflanzliche Rohstoffe wurden zu neuartigen Lebensmitteln verarbeitet [3]. Es entstand eine duale diätetische Strategie, bei der eine überwiegend pflanzliche Kost um Fleischmahlzeiten ergänzt wurde [4].

Der vor 200000 Jahren erscheinende moderne Mensch (Homo sapiens) verbreitete sich von seinem Entwicklungszentrum in Afrika weltweit aus. Mit der neolithischen Revolution kam es schliesslich zu den grundlegenden Umstellungen der Ernährung, wie wir sie bis heute kennen $[5,6]$.

Allen Veränderungen der Ernährung seit dem Neolithikum zum Trotz definiert das evolutionäre Erbe einer Adaptation der Hominiden an eine vielfältige Pflanzenkost weiterhin auch für den modernen Menschen den Umfang und die Grenzen der metabolischen Verwertbarkeit von Pflanzen. So bleibt z.B. die Verdauung von Zellulose unmöglich [7].

\section{Koevolution von Pflanzen und Tieren}

Pflanzen sind als autotrophe Organismen die Basis unserer Existenz; sie wandeln physikalische Sonnenenergie in organische Substanz um, die am Anfang der biologischen Stoffkreisläufe steht.

Tierisches Leben befindet sich in viel offenkundigerer Abhängigkeit von Pflanzen, als dies in umgekehrter Richtung der Fall zu sein scheint. Es bestehen jedoch wechselseitige Abhängigkeiten, welche die Diversität der Pflanzen und ihre individuelle Komplexität erst erklären. Interaktionen von Pflanzen mit der Tierwelt sind in besonderer Weise für die Insekten aufgeklärt.

Fremdbestäubte Pflanzen bedienen sich herbivorer Arthropoden für ihre sexuelle Fortpflanzung. Duftstoffe, Nektar und/oder nahrhafte Pollen locken Insekten in das Zentrum ihrer Blüten, wo diese bei der Nahrungsaufnahme zwangsläufig die Befruchtung vollziehen. Die Symbiosen von Pflanzen mit bestäubenden Insekten haben sich über Generationen der Koevolution vervollkommnet und haben als Ko-Speziation zur Artbildung geführt. Der Begriff der Koevolution wurde erstmals 1958 von Mode [8] eingeführt und später von Ehrlich und Raven [9] weiterentwickelt.

In minutiöser wechselseitiger Anpassung liess die Koevolution aufeinander geprägte Artenpaare entstehen. Die unfehlbare gegenseitige Abstimmung nach dem Schlüssel-Schloss-Prinzip liess Charles Darwin für Madagaskar einen Schmetterling mit ca. $20 \mathrm{~cm}$ langem Rüssel (Proboscis) prognostizieren, weil die madegassische Orchidee (Angraecum eburneum) mit entsprechend langem Blütensporn dessen Existenz erforderlich machte. Tatsächlich wurde dieser Nachtfalter Jahrzehnte später auf Madagaskar entdeckt. Der Falter Xanthopan morganii erhielt zur Erinnerung an Darwins Voraussage den Beinamen «praedicta».

\section{Die sogenannten pflanzlichen Sekundärstoffe}

Pflanzen versichern sich der Anwesenheit ihrer bestäubenden Insekten, indem sie ihnen beispielsweise die Eiablage ermöglichen und sich als Futter der Raupen darbieten. Gleichzeitig wehren sie Insekten und andere Organismen ab, die ihnen Schäden zufügen könnten.

Für den jeweiligen Zweck biosynthetisieren sie sogenannte pflanzliche Sekundärstoffe. In Symbiose lebende Insekten erkennen spezifische Sekundärstoffe als Lockstoffe, während die Imagines oder Larven von Schadinsekten dagegen durch andere Sekundärstoffe abgeschreckt oder vergiftet werden. Symbiontische Insekten schirmen ihre Vitalfunktionen ab, indem sie die Toxine in am Stoffwechsel unbeteiligten Geweben sequestrieren. 
Mitunter müssen sich die Pflanzen selbst vor den eigenen biosynthetisierten Toxinen schützen. Dies gelingt ihnen ebenfalls durch eine Kompartimentalisierung oder indem sie die Substanzen als untoxische Vorstufen vorhalten. Erst bei einer Verletzung der Pflanzen werden in Vakuolen gespeicherte cyanogene Glykoside zu Blausäure und Senfölglykoside zu Thiocyanaten «scharf geschaltet» [10]. Die Sekundärstoffe sichern den Pflanzen das Überleben in einer feindlichen Umwelt, was den Aufwand ihrer Biosynthese erklärt.

Der Antagonismus der pflanzlichen Sekundärstoffe mit den korrespondierenden Schutzmechanismen aufseiten der Tiere ist ein phylogenetisch altes Prinzip, das sich seit 200 Millionen Jahren der Koevolution beständig erneuert. Diejenigen Pflanzen überleben, die der entstehenden Toleranz der Insekten gegenüber ihren Toxinen mit immer stärkeren, neuartigen und höher konzentrierten Toxinen begegnen. Auf diese Weise entstand nach heutiger Überzeugung die hohe Diversität sekundärer Pflanzenstoffe in der rezenten Flora.

Nach ihrer Entdeckung galten die Sekundärstoffe zunächst als funktionslos und als blosse Abfallprodukte des pflanzlichen Stoffwechsels.

Fraenkel [11] entwickelte 1959 erstmals die Vorstellung, dass sie eine spezifische Funktion ausüben könnten. Harborne [12] klassifiziert die Sekundärstoffe nach ihrer chemischen Struktur grob in Phenole, N-haltige Verbindungen und Terpenoide. Die Wirkung der Sekundärstoffe korreliert grundsätzlich nicht mit ihrer Konzentration oder dem Gesamtgehalt in den Pflanzen, sondern sie ist das Resultat einer passgenauen Interaktion mit TargetStrukturen auf der Seite des Konsumenten. So können auch sehr geringe Mengen einzelner Sekundärstoffe für gravierende Wirkungen beim Menschen sorgen. Im Verlauf von unzähligen Generationen entstand die rezente Palette der Sekundärstoffe als biologisch wirksame Moleküle durch Interaktion mit korrespondierenden Targets der tierischen Organismen - man spricht vom evolutionären molekularen Modelling.

Angesichts der geradezu verschwenderischen strukturellen Diversität und Vielzahl der Sekundärstoffe stellt sich die Frage, wie sich Pflanzen den biosynthetischen Aufwand für Moleküle mit begrenzten Sonderaufgaben leisten können. Dieser Aufwand wird möglicherweise überschätzt. Inzwischen konnte experimentell rekapituliert werden, wie sich diese relativ kleinen Moleküle über nur wenige enzymatische Umwandlungen aus dem Primärstoffwechsel der Pflanzen abzweigen lassen [13].

\section{Sensorische Wahrnehmung der Sekundärstoffe}

Der frühe Mensch musste sich mit den Sekundärstoffen einer Vielzahl von Pflanzen auseinandersetzen, um sie als potenzielle Nahrungsquellen $\mathrm{zu}$ bewerten. Toxische Substanzen galt es zu meiden. Zu ihrer Erkennung verliess er sich wie die Tiere auf sein Sensorium. Dabei ist der Umstand, dass sich viele Toxine durch sensorisch deutlich wahrnehmbare Signale erkennen lassen, von lebenswichtiger Bedeutung. Geschmack und Aussehen von Pflanzen zeigen häufig gleichsinnig eine Gefahr und Giftigkeit für Tiere an, die bittere oder scharf schmeckende Früchte bereits aufgrund ihrer Warnfarben (aposematische Färbung) instinktiv meiden. Der Begriff der aposematischen Färbung wurde zunächst für das Tierreich eingeführt. Diese Färbung schützt die Tiere vor möglichen Prädatoren, indem sie auf eine vorhandene Wehrhaftigkeit oder Ungeniessbarkeit hinweist.

Für Pflanzen wurde lange Zeit verkannt, dass das Prinzip der aposematischen Warnfarben hier ebenso gilt, obwohl bereits bekannt war, dass auch die Farbwirkung der Tiere auf eingelagerten pflanzlichen Pigmenten beruht. Verbreitet wird eine weisse, gelbe, orange oder braune aposematische Färbung von bewehrten (Dornen, Stacheln usw.), toxischen oder ungeniessbaren Pflanzen präsentiert. An Früchten, die lange vor ihrer Reife bereits ihre volle Grösse erreicht haben, wird die Ungeniessbarkeit mit genau diesen Farben signalisiert, bevor die reifen Früchte nochmals zu ihrer endgültigen Farbe umschlagen. In allen Klimazonen einschliesslich der Tropen sind Schwarz und Rot die häufigsten Farben reifer Früchte. Ähnlich wie im Tierreich gibt es unter Pflanzen eine blosse Vortäuschung einer Wehrhaftigkeit durch die Präsentation einer aposematischen Färbung (Phänomen der Mimikry) $[14,15]$.

Eine verbreitete Klassifizierung der Qualität der chemischen Abwehr von Pflanzen (nach Harborne [12]) teilt diese in Bitterstoffe, Toxine, Stoffe mit unangenehmem Geruch und solche mit antinutritiver Wirkung ein. Menschen bewerten süsse und fettreiche Nahrungsquellen als wohlschmeckend, während sie Bitterstoffe meiden. Mit diesen Geschmackspräferenzen greifen Menschen instinktiv auf Nahrungsmittel mit einem hohen Nährstoffgehalt für sie zurück.

Bitterstoffe in Pflanzen bewahren den Menschen vor der Aufnahme grösserer Mengen und verhindern Vergiftungen bei Kindern, z.B. im Fall von Fingerhut oder Maiglöckchen. Der in allen Teilen giftige Attich (Sambucus ebulus) hat einen so abstossenden Geruch, dass selbst die verführerisch glänzenden schwarzen Früchte kaum konsumiert werden dürften [16].

Das menschliche Sensorium ist allerdings unvollkommen und hat gegenüber anderen Primaten wie den Schimpansen oder den ausgestorbenen Neandertalern oder Denisova-Menschen an Trennschärfe eingebüsst. Untersuchungen des Genoms dieser nahe verwandten Primaten zeigen, dass jene noch über Rezeptoren zur Erkennung von Bitterstoffen verfügten, die dem moder- 


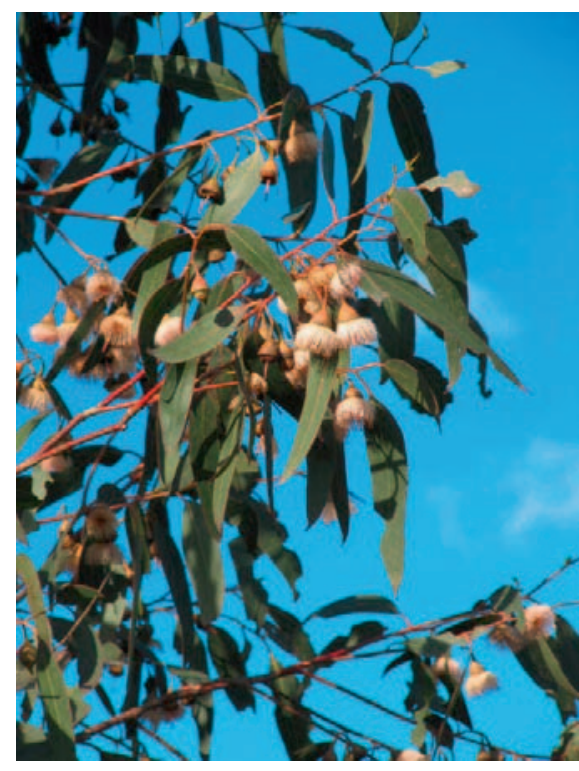
Eucalyptus globosus bei Melbourne in Australien, 2011.
Abb. 2. Ein Koala (Phascolarctos cinereus) ruht in einer Astgabel von Eucalyptus spec. in der Nähe von Melbourne, 2011.

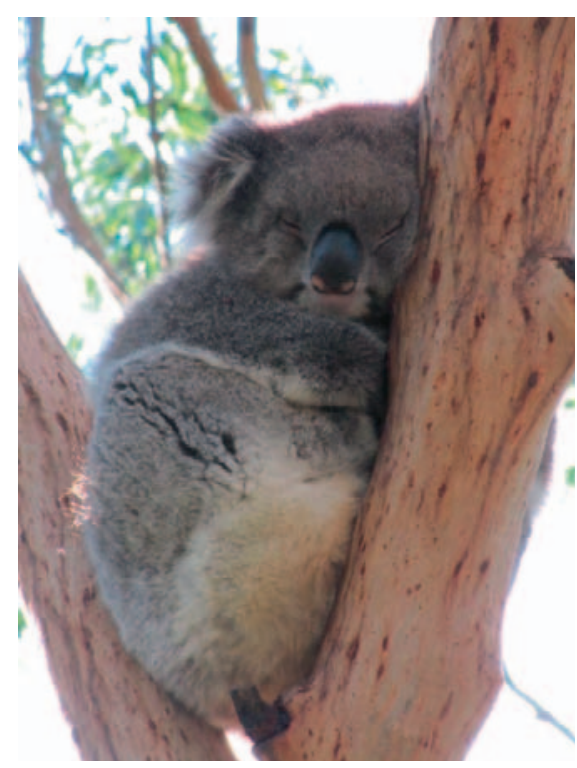

nen Menschen fehlen [17]. Der moderne Mensch ist zum sensorischen Krüppel geworden und setzt bei der Bewertung von Nahrung auf eine zivilisatorische Informationsweitergabe.

Zusätzlich bestehen interindividuelle und interkulturelle Unterschiede bezüglich der sensorischen Kompetenz des Menschen. Geschmäcker und Gerüche werden nicht einheitlich von Menschen bewertet. So verströmen die Früchte des Durianbaums (Durio zibethinus) durch Dithiohalbacetale einen Geruch, der von vielen als widerwärtig empfunden wird. Andere finden die Aromen delikat und betrachten die Frucht als Delikatesse. Auch in der Bewertung des Geruchs des Rainfarns (Tanacetum vulgare) sind die Menschen sich uneins - erst in jüngster Zeit wurde entdeckt, dass die vor allem im Mittelalter genutzte Heilpflanze tödliche Vergiftungen verursachen kann, wovor der Geruch vielleicht warnen soll. Die Bitterkeit des 6-n-Propylthiouracils (PROP) wird nur von einem Teil der Menschen geschmeckt. Die PROP-Schmecker nehmen die bitteren Sekundärstoffe in vielen Gemüsen besonders intensiv wahr. Sie meiden Gemüse, ganz besonders während der Kindheit [18]. In Kenntnis dieses Zusammenhangs sollte man Nachsicht mit Kleinkindern haben, die sich einem elterlich verordneten Zwang zum Gemüsekonsum widersetzen.

Heute setzt sich die moderne Menschheit nicht mehr mit einer unbekannten Pflanzenwelt auseinander, um sich von ihr zu ernähren. Vor wenigen Jahrhunderten noch erkundeten Europäer fremde Landstriche und mussten mithilfe von Geschmacksproben abwägen, welche Pflanzen zur Ernährung geeignet waren. Die Routen der grossen Entdeckungsreisen im 18. und 19. Jahrhundert führten auch zu unbewohnten subantarktischen Inseln im Indischen Ozean, unter anderem zur Gruppe der Kerguelen. Dort trafen die Seeleute auf eine Pflanze, die sie nach Aussehen und Geschmack an heimischen Kohl erinnerte und die sie als Speise erprobten. Die Kerguelenkohl (Pringlea antiscorbutica) genannte Pflanze war später in der Seefahrt wegen ihres Gehalts an Vitamin C zur Verhinderung des Skorbuts geschätzt.

Nicht alle problematischen Sekundärstoffe können sensorisch als Gefahr eingeordnet werden. So wird eine gestörte Verfügbarkeit wichtiger Nährstoffe durch antinutritive Substanzen erst verzögert als Folge einer Langzeitexposition bemerkt. Das gilt für die sogenannten Goitrogene des Kohls, welche die Jod-Aufnahme hemmen und zur Entwicklung einer Struma führen [19]. Phytinsäure aus Getreide oder Oxalsäure im Spinat können die Eisenaufnahme des Organismus stören und so einen Eisenmangel verursachen. Der vergleichsweise noch geringe Oxalsäuregehalt im Spinat alarmiert das menschliche Sensorium nicht [20].

\section{Toxische Sekundärstoffe werden unschädlich gemacht}

Die Vermeidung aller Pflanzen mit problematischen Sekundärstoffen würde das Nahrungsangebot für den Menschen einschränken und einen Evolutionsnachteil darstellen. Sowohl die Tiere als auch der Mensch haben daher detoxifizierende Mechanismen (Transporter, Enzyme und andere) entwickelt, die es ihnen erlauben, sich von einem breiteren Spektrum an Pflanzen mit primär problematischen Inhaltsstoffen zu ernähren.

Ein eindrucksvolles Beispiel ist der australische Koala (Phascolarctos cinereus). Er frisst ausschliesslich Blätter von Eucalyptus-Bäumen (Abb. 1, 2). Die in den Blättern konzentrierten ätherischen Öle sind für Koalas giftig. Dennoch gelingt es dem Beuteltier durch einen speziell 


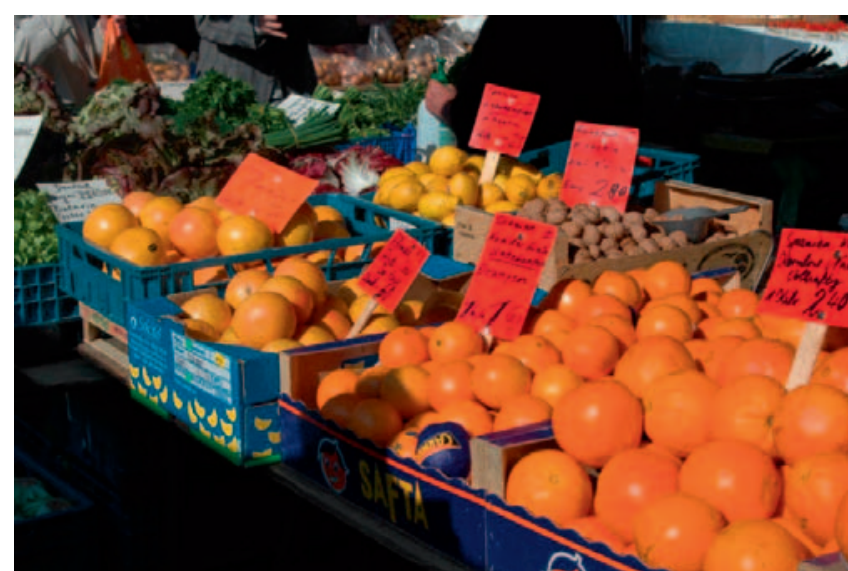

Abb. 3. Zitrusfrüchte, speziell die Grapefruit (Citrus $\times$ aurantium), beein flussen den Medikamentenstoffwechsel durch Hemmung des CytochromP450-Enzyms CYP3A4.

angepassten Verdauungsprozess, den Blättern Nährstoffe und Wasser zu entziehen, während die Toxine in seinem langen Blinddarm durch Mikroben zersetzt werden. $\mathrm{Zu}$ sätzlich regulieren Koalas die Toxinzufuhr, indem sie bevorzugt die älteren Blätter mit einem geringeren Toxingehalt fressen.

Der tropische Käfer Caryedes brasiliensis ernährt sich von den Samen der Leguminose Dioclea megacarpa und reichert die enthaltene toxische nichtproteinogene Aminosäure L-Canavanin an. Die zwingende Toxizität von L-Canavanin beruht darauf, dass sie bei der Proteinbiosynthese anstatt des L-Arginins in Proteinen inkorporiert wird und strukturell defekte Moleküle entstehen lässt. Die Larve von C. brasiliensis vermeidet dies durch eine spezielle hochdiskriminatorische Arginin-tRNA-Ligase, die den ersten Schritt der Arginin-Inkorporation katalysiert und L-Canavanin zurückweist. Für alle anderen Organismen sind die Samen von Dioclea und sekundär auch der Käfer C. brasiliensis aufgrund des Gehalts an L-Canavanin toxisch [21].

Transportproteine wie das p-Glykoprotein (P-GP) und die zahlreichen Isoformen der Cytochrom-P450(CYP)Enzyme ermöglichen dem tierischen Organismus die kontrollierte Aufnahme pflanzlicher Allelochemikalien und deren Entgiftung. Dies erfolgt entweder durch unmittelbare Wiederausschleusung über Transporter oder durch Metabolisierung zu nichttoxischen Zwischenformen und deren sekundäre Elimination über die Galle oder den Urin. Erkenntnisse zur Substratspezifität und -kinetik dieser Mechanismen erhielt die Forschung durch Markersubstanzen wie chemisch definierte Arzneistoffe, die sich diese Abbauwege mit den Naturstoffen teilen. An den Bindungsstellen der Transporter und CYP-Enzyme konkurrieren Medikamente und Naturstoffe miteinander und regulieren dadurch ihre Wirkspiegel in gegensinniger Weise [22].

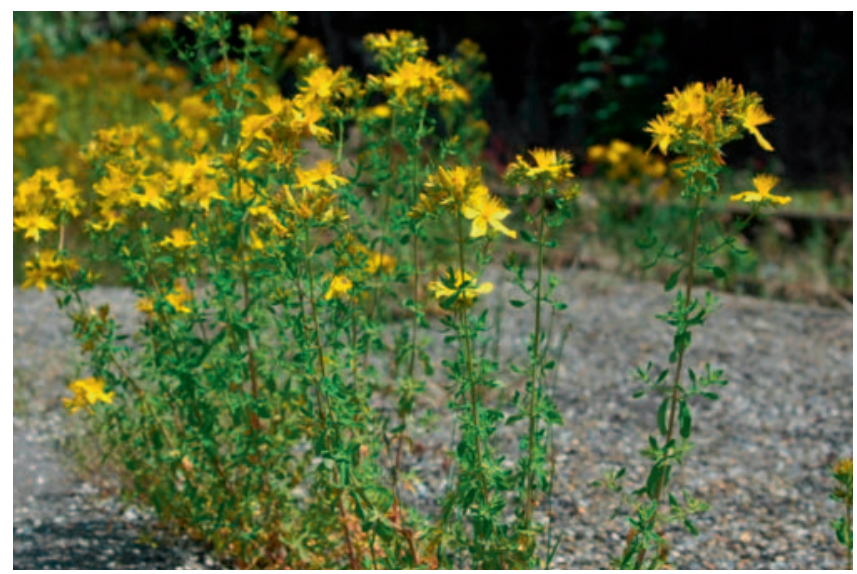

Abb. 4. Johanniskraut (Hypericum perforatum) enthält antidepressive Wirkstoffe. Es stimuliert ausserdem die Aktivität des Enzyms CYP3A4, über das viele Arzneimittel abgebaut werden.

Viele Naturstoffe sind zudem in der Lage, die Gewebeexpression und die Aktivität der Detoxifikationsmechanismen zu modulieren. Beispiele hierfür sind die Hemmung der Aktivität des Isoenzyms CYP3A4 durch die Furanocumarine der Grapefruit (Abb. 3) [23] und seine Überexpression und Aktivitätssteigerung durch Wirkstoffe des Johanniskrauts (Abb. 4) [24, 25]. Durch Veränderungen der Aktivität solcher CYP-Isoenzyme, die an der Entstehung kanzerogener Naturstoffe beteiligt sind, versucht man eine epidemiologisch nachweisbare Krebsprotektion durch eine an Gemüsen wie Kohl und Knoblauch reiche Ernährung zu erklären.

Die CYP-Enzyme gehören dem Phase-I-Metabolismus an, mit dem aufgenommene Pflanzenstoffe wie die hydrophoben Alkaloide, Terpene, Terpenoide und Furanocumarine durch Oxidation wasserlöslich gemacht werden, um dann weiteren Prozessen wie der Konjugation usw. zugeführt zu werden [26]. Eine andere Möglichkeit für den tierischen Organismus, sich pflanzlicher Allelochemikalien zu entledigen, ist der Abbau durch mikrobielle Symbionten (wie im Beispiel des Koala).

Die Leistungsfähigkeit der detoxifizierenden Enzyme beim Menschen weist ethnische und geographische Unterschiede auf. Beispiele hierfür sind die Verdauungskapazität für Leguminosen-Proteine oder die Aufnahme und der Abbau von Arzneimitteln wie dem Paracetamol, die bei Europäern und Asiaten unterschiedlich rasch ablaufen [27].

Die in einem hohen Masse auf ihre Substrate spezialisierten CYP-Isoenzyme sind in Bevölkerungsgruppen unterschiedlich repräsentiert, was auf einer historisch vorherrschenden Exposition gegenüber bestimmten pflanzlichen Nahrungsmitteln zu beruhen scheint. Indirekt erklärt dies die genetischen Polymorphismen für die Metabolisierungskapazität bestimmter Arzneimittel wie dem in der Transplantationsmedizin bedeutsamen Cyclosporin A [28]. Unterschiede im Cyclosporin-Metabolismus, 
wie sie zwischen Amerikanern afrikanischer Herkunft und solchen mit europäischen Vorfahren bestehen, erklären einen voneinander abweichenden Dosisbedarf für das Erreichen der Zielblutspiegel [29]. Diese Befunde untermauern das Konzept einer geographisch und genetisch gebundenen Prägung des Detoxifikationsarsenals innerhalb bestimmter Bevölkerungsgruppen als Resultat der Koevolution mit der lokalen Pflanzenwelt [30].

\section{Instrumentalisierung von Sekundärstoffen: Beispiele und Modelle für Krankheitsinduktion und -protektion}

Herbivoren vermeiden oder entschärfen toxische Sekundärstoffe nicht nur, sondern sie können diese auch für ihre eigenen Zwecke nutzen. Im einfachsten Fall geschieht dies, wenn Insekten die mit der Pflanzennahrung aufgenommenen Moleküle unverändert als «borrowed molecules» durch ihren Körper schleusen und zur Abwehr von Prädatoren wieder abgeben. Bekannt ist dies von Heuschrecken (Poikilocerus bufonius), die sich auf ihren Futterpflanzen der Gattung Asclepias mit Terpenoiden (toxische Cardenolide) aufladen, um diese bei einer Attacke durch Vögel auszuscheiden [31].

Raffinierter ist die Rolle der toxischen Alkaloide der Gattung Parsonsia aus der Familie der Hundsgiftgewächse (Apocynaceae) für den Schmetterling Weisse Baumnymphe (Idea leuconoe) auf den Philippinen. Die Raupen ernähren sich von den Parsonsia-Blättern. Die Weibchen erkennen die zur Eiablage geeigneten Pflanzen am Gehalt der für Wirbeltiere hochgiftigen Pyrrolizidinalkaloide Parsonsianin, Parsonsianidin und 17-Methylparsonsianidin. Die Raupen und die Imagines können diese toxischen Alkaloide in grosser Menge speichern und sich damit vor Prädatoren schützen. Mehr noch: Die Schmetterlinge bilden durch biochemische Umwandlung aus den Alkaloiden die Pheromone Viridifloric $\beta$-Lacton und Danaidon, mit denen die Männchen die Weibchen anlocken [32]. Die toxischen Sekundärstoffe werden also in mehrfacher Hinsicht instrumentiert und erhalten eine Schlüsselrolle im Entwicklungszyklus der Insekten.

Dies ist nur eines von vielen weiteren Beispielen einer raffinierten Instrumentalisierung von Sekundärstoffen durch Insekten. Kommen wir noch einmal zum Käfer $C$. brasiliensis und seiner Ausschaltung der obligat toxischen Aminosäure L-Canavanin zurück. Diese ist auch für den Menschen gefährlich. Über die akute Toxizität durch den Ausfall lebenswichtiger, in ihrer tertiären und quartären Proteinstruktur gestörten Enzyme hinaus könnten diese Konformationsänderungen ein Modell für die Entwicklung von Autoimmunkrankheiten bei Primaten sein. Die in ihrer Struktur veränderten Proteine werden vom Immunsystem als «Nicht-Selbst» verkannt und induzieren

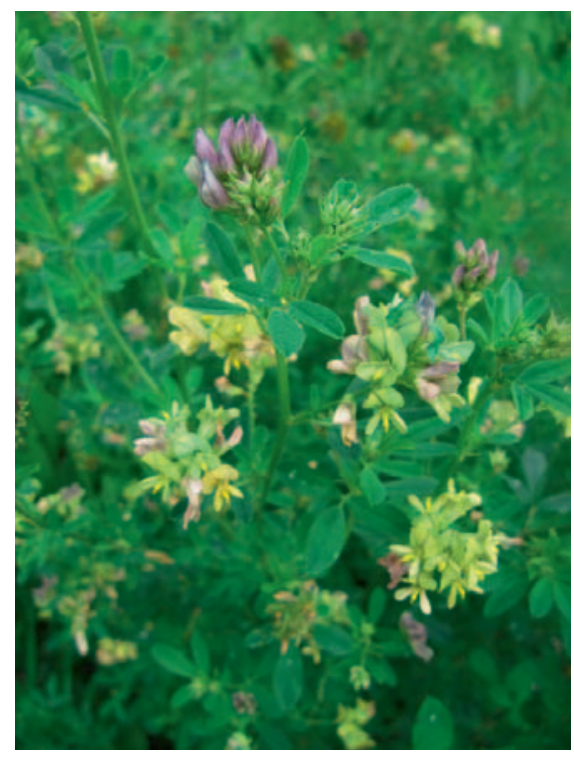

die Produktion von Autoantikörpern. Nachdem auch Samen und Sprossen von Alfalfa oder Luzerne (Medicago sativa) L-Canavanin enthalten und die Sprossen gerne im Salat verspeist werden, ist eine Exposition beim Menschen nicht ausgeschlossen (Abb. 5). Experimentell löst die Aufnahme von Alfalfa-Sprossen bei Primaten eine dem systemischen Lupus erythematodes (SLE) ähnliche Autoimmunerkrankung und weitere Autoimmunphänomene aus. Die Autoimmunerkrankungen kommen zum Stillstand, sobald die Alfalfa-Aufnahme unterbleibt [33]. Die Bedeutung dieser interessanten Beobachtungen für die ungeklärte Ätiologie vieler Autoimmunerkrankungen und Kollagenosen ist wissenschaftlich nicht ausreichend aufgearbeitet. Gibt es einen Zusammenhang der Prävalenz von SLE mit einer regionalen Bevorzugung bestimmter Leguminosen in der Ernährung? Neben einer gut belegten genetischen Prädisposition für SLE werden externe Auslöser für die Erkrankungsschübe angenommen. Die genetische Prädisposition könnte Unterschiede in der Verarbeitung dieser externen Stimuli reflektieren.

Die Entschärfung primär toxischer Pflanzenprodukte und ihre nachfolgende Instrumentalisierung durch Insekten stehen modellhaft für eine pharmakologische Nutzung von Pflanzen durch den Menschen zur Prophylaxe und Therapie von Krankheiten.

Pflanzliche Sekundärstoffe in der Nahrung können die Aktivität von Transportern und CYP-Enzymen modulieren. Am Modell der Bioverfügbarkeit bestimmter Medikamente wurde bereits der Einfluss von Furanocumarinen der Grapefruit oder der Wirkstoffe des Johanniskrauts auf CYP3A4 geschildert. Vergleichbare Folgen können für weitere Naturstoffe eintreten, die über dieses Isoenzym metabolisiert werden. Ihre Bioverfügbarkeit im Organismus kann sinken oder steigen - je nachdem, wie die Aktivität der CYP-Enzyme verstellt wird. 
Einige CYP-Isoenzyme, wie z.B. das CYP2E1, katalysieren für den Menschen ungünstige Reaktionen, durch die Kanzerogene erst aktiviert werden. Diallylsulfide des Knoblauchs (Allium sativum) hemmen die Aktivität von CYP2E1, was eine epidemiologisch und tierexperimentell vermutete kanzeroprotektive Wirkung des Knoblauchs erklären könnte. Broccoli (Brassica oleracea var. italica) dagegen vermag, wie vermutlich andere Cruciferen auch, bestimmte Phase-II-Detoxifikationsenzyme, z.B. die Quinon-Reduktase oder die Glutathion-S-Transferase, zu induzieren und damit Schutz vor Kanzerogenen sowie vor oxidativen Schäden zu vermitteln [34, 35].

Im Modell einer genetischen Hypertonie bei Ratten führt Sulforaphan, das wichtigste aktive Isothiocyanat in Cruciferen-Gemüse, zur Hemmung von CYP4A, das die Degradierung der vasodilatatorischen Prostanoide in der Niere steuert. Sulforaphan senkt in diesem Modell den Blutdruck [36].

Es gilt heute als gesichert, dass der Mensch die antibakteriell wirksamen Sekundärstoffe der Pflanzen frühzeitig zu nutzen begann, indem er verderbliches Fleisch mit Kräutern und Gewürzen konservierte. Die Inhaltsstoffe waren zusätzlich geschmacksverbessernd und konnten nach ihrer Aufnahme mit der Fleischmahlzeit intern weiterwirken, z.B. zur Bekämpfung vorliegender Infektionen. Wie antimikrobielle Wirkstoffe aus Pflanzen resorbiert werden und über die Blutzirkulation an ihre Wirkorte gelangen, ist für das Arbutin aus den Blättern der Bärentraube (Arctostaphylos uva-ursi) [37] sowie für die Typ-A-Proanthocyanide aus der Cranberry (Vaccinium macrocarpon) und ihre Wirksamkeit bei Harnwegsinfektionen sehr genau untersucht [38, 39].
Solange der Mensch omnivorer Jäger und Sammler war, hat er vermutlich hinreichende Mengen dieser Sekundärstoffe mit einer vielfältigen pflanzlichen Ernährung aufgenommen. Dieser Vorteil der ursprünglichen Ernährungsweise musste mit dem einseitigen Verzehr weniger domestizierter Pflanzen verloren gehen. Mangelerscheinungen und Gesundheitsrisiken durch die fehlende Zufuhr von Sekundärstoffen traten somit in Erscheinung.

\section{Fazit und Ausblick auf die Fortsetzungen}

Die Bedeutung der Sekundärstoffe für die Nahrungsauswahl des Ur-Menschen macht eine mitentscheidende Rolle dieser Moleküle für die Auswahl der Pflanzen zur Domestikation wahrscheinlich. Im zweiten Beitrag, «Bedeutung der Sekundärstoffe bei der Domestikation der Nahrungspflanzen», wird dies am Beispiel von Nüssen und den «founder crops» der Getreide und Leguminosen und weiteren Pflanzen untersucht. Der dritte und letzte Beitrag, «Gemüse, Gewürze und Arzneipflanzen als Quelle wertvoller Sekundärstoffe», hat die von den Grundnahrungsmitteln abgekoppelte Versorgung mit den für uns nützlichen Sekundärstoffen aus Pflanzen zum Thema.

\section{Disclosure Statement}

Keine Interessenkonflikte.

\section{Literatur}

$>_{1}$ White TD, Asfaw B, Beyene Y, Haile-Selassie Y, Lovejoy CO, Suwa G, Wolde GG: Ardipithecus ramidus and the paleobiology of early hominids. Science 2009;326:75-86.

$\checkmark 2$ Teaford MF, Ungar PS: Diet and the evolution of the earliest human ancestors. Proc Natl Acad Sci U S A 2000;97:13506-13511.

$\checkmark 3$ de Heinzelin J, Clark JD, White T, Hart W, Renne P, Wolde-Gabriel G, Beyene Y, Vrba E: Environment and behaviour of 2.5-million-year-old Bouri hominids. Science 1999; 284:625-629.

4 Milton K: The critical role played by animal source foods in human (Homo) evolution. J Nutr 2003; 133:S3886.

5 Ye K, Gu Z: Recent advances in understanding the role of nutrition in human genome evolution. Adv Nutr 2011;2:486-496.

6 Diamond J: Evolution, consequences and future of plant and animal domestication. Nature 2002;418:700-707.
Krebs JR: The gourmet ape: evolution and human food preferences. Am J Clin Nutr 2009;90(suppl):707S-711S.

8 Mode CJ: A mathematical model for the coevolution of obligate parasites and their hosts. Evolution 1958;12:158-165.

$\checkmark 9$ Ehrlich PR, Raven PH: Butterflies and plants: a study in coevolution. Evolution 1964;18: 586-608.

10 Sitte P, Weiler EW (Hrsg): Strasburger. Lehrbuch der Botanik, ed 35. Heidelberg, Spektrum, 2002.

11 Fraenkel GS: The raison d'etre of secondary plant substances. Science 1959;129:1466-1470.

12 Harborne JB: Introduction to Ecological Biochemistry. New York, Academic Press, 1994.

13 Storch V, Welsch U, Wink M: Evolutionsbiologie, ed 2. Heidelberg, Springer, 2007.

14 Lev-Yadun S: Plant-environment interactions: aposematic (warning) coloration in plants; in Baluska F (ed): Signaling and Communication in Plants. Heidelberg, Springer, 2009, pp 167-202.
15 Lev-Yadun S, Ne’eman G, Izhaki I: Unripe red fruits may be aposematic. Plant Signal Behav 2009;4:836-841.

16 Nowack R: Notfallhandbuch Giftpflanzen. Ein Bestimmungsbuch für Ärzte und Apotheker. Berlin/Heidelberg, Springer, 1998.

17 Choi CQ: Different Tastes: How Our Human Ancestors Diets Evolved. Live Science, January 16, 2015. www.livescience.com/49477human-ancestors-diet-taste-evolution.html.

18 Bell KI, Tepper BJ: Short-term vegetable intake by young children classified by 6 -n-propylthoiuracil bitter-taste phenotype. Am J Clin Nutr 2006;84:245-251.

19 Greer MA: Goitrogenic substances in food. Am J Clin Nutr 1957;5:440-444.

20 Zhou JR, Erdman JW Jr: Phytic acid in health and disease. Crit Rev Food Sci Nutr 1995;35: 495-508.

21 Rosenthal GA, Hughes CG, Janzen DH: LCanavanine, a dietary nitrogen source for the seed predator Caryedes brasiliensis (Bruchidae). Science 1982;217:353-355. 
22 Nowack R: Review article: cytochrome P450 enzyme, and transport protein mediated herb-drug interactions in renal transplant patients: grapefruit juice, St John's Wort and beyond! Nephrology (Carlton) 2008;13: 337-347.

-23 Nowack R, Andrassy J, Fischereder M, Unger M: Effects of dietary factors on drug transport and metabolism: the impact on dosage guideline in transplant patients. Clin Pharmacol Ther 2009;85:439-443.

$\checkmark 24$ Breidenbach T, Kliem V, Burg M, Rader macher J, Hoffmann MW, Klempnauer J: Profound drop of cyclosporin A whole blood trough levels caused by St. John's wort (Hy pericum perforatum). Transplantation 2000 69:2229-2230.

25 Dürr D, Stieger B, Kullak-Ublick GA, et al: St. John's wort extract induces intestinal P-gly coprotein/MDR1 and intestinal and hepatic cytochrome CYP3A4 in man. Clin Pharmacol Ther 2000;68:598-604.

26 Herrera CM, Pellmyr O (eds): Plant-Anima Interactions. An Evolutionary Approach. Oxford, Blackwell, 2002.

-27 Lamba JK, Lin YS, Schuetz EG, Thummel KE: Genetic contribution to variable human CYP3A-mediated metabolism. Adv Drug Deliv Rev 2002;54:1271-1294.
28 Wandel C, Witte JS, Hall JM, Stein CM, Wood AJ, Wilkinson GR: CYP3A activity in African American and European American men: population differences and functional effect of the CYP3A $4^{\star} 1 \mathrm{~B} 5{ }^{\prime}$-promoter region polymorphism. Clin Pharmacol Ther 2000 68:82-91.

29 Crettol S, Venetz JP, Fontana M, Aubert JD, Pascual M, Eap CB: CYP3A7, CYP3A5, CYP3A4, and ABCB1 genetic polymorphisms, cyclosporine concentration, and dose requirement in transplant recipients. Ther Drug Monit 2008;30:689-699.

30 Dirks NL, Huth B, Yates CR, Meibohm B: Pharmacokinetics of immunosuppressants: a perspective on ethnic differences. Int J Clin Pharmacol Ther 2004;42:701-718.

31 Rothschild M, Kellett DN: Notes on the reactions of various predators to insects storing heart poisons (cardiac glycosides) in their tissues. J Entomol Ser A 1972;46:103-110.

32 Honda K, Hayashi N, Abe F, Yamauchi T: Pyrrolizidine alkaloids mediate host-plant recognition by ovipositing females of an Old World danaid butterfly, Idea leuconoe. Chem Ecol 1997;23:1703-1713.

33 Malinow MR, Bardana EJ Jr, Pirofsky B, Craig S, McLaughlin P: Systemic lupus erythematosus-like syndrome in monkeys fed alfalfa sprouts: role of a nonprotein amino acid. Science 1982;216:415-417.
34 Pantuck E, Pantuck C, Garland W, et al: Stimulatory effect of brussels sprouts and cabbage on human drug metabolism. Clin Pharmacol Ther 1979;25:88-95.

35 Zhang Y, Talalay P, Cho CG, Posner GH: A major inducer of anticarcinogenic protective enzymes from broccoli: isolation and elucidation of structure. Proc Natl Acad Sci U S A 1992;89:2399-2403.

36 Elbarbry F, Vermehren-Schmaedick A, Balkowiec A: Modulation of arachidonic acid metabolism in the rat kidney by sulforaphane: implications for regulation of blood pressure. ISRN Pharmacol 2014;2014:683508.

37 Schindler G, Patzak U, Brinkhaus B, von Niecieck A, Wittig J, Krähmer N, Glöckl I, Veit M: Urinary excretion and metabolism of arbutin after oral administration of Arctostaphylos uvae ursi extract as film-coated tablets and aqueous solution in healthy humans. J Clin Pharmacol 2002;42:920-927.

38 Nowack R: Die amerikanische Cranberry Portrait einer Arzneipflanze. Z Phytother 2003;24:40-46.

39 Nowack R, Birck R: Cranberry products in the prevention of urinary tract infections: examining the evidence. Botanics: Targets and Therapy 2015;5:45-54. 